\title{
Magnetic measurements on micron-size samples under high pressure using designed NV centers
}

\author{
Margarita Lesik ${ }^{1 *}$ Thomas Plisson ${ }^{2, * \dagger}$, Loïc Toraille ${ }^{1, *}$, Justine Renaud $^{3}$, \\ Florent Occelli ${ }^{2}$, Martin Schmidt ${ }^{1}$, Olivier Salord ${ }^{3}$, Anne Delobbe ${ }^{3}$, \\ Thierry Debuisschert ${ }^{4}$, Loïc Rondin ${ }^{1}$, Paul Loubeyre ${ }^{2}$, and Jean-François Roch ${ }^{1, \dagger}$ \\ ${ }^{1}$ Laboratoire Aimé Cotton, CNRS, Univ. Paris-Sud, ENS Cachan, \\ Université Paris-Saclay, 91405, Orsay Cedex, France \\ ${ }^{2}$ CEA, DAM, DIF, 91297 Arpajon, France \\ ${ }^{3}$ Orsay Physics S. A., 95 avenue des Monts Auréliens, 13710 Fuveau, France \\ ${ }^{4}$ Thales Research \& Technology, 1, avenue Augustin Fresnel, 91767 Palaiseau, France \\ ${ }^{\dagger}$ To whom correspondence should be addressed; \\ E-mail: thomas.plisson@ cea.fr, jean-francois.roch@ens-paris-saclay.fr.
}

Pressure is a unique tool to tune the interplay between structural, electronic and magnetic interactions. It leads to remarkable properties of materials such as recent temperature records in superconductivity. Advanced magnetic measurements under very high pressure in the Diamond Anvil Cell (DAC) use synchrotron approaches but these are lacking a formal link to the macroscopic magnetic properties. We report an alternative method consisting in optical magnetometry based on nitrogen-vacancy (NV) centers created at the surface of a diamond anvil. We illustrate the method by two measurements realized at room and low temperature respectively: the pressure evolution of the magnetization of an iron bead up to $30 \mathrm{GPa}$ showing the iron ferromagnetic collapse and the detection of the superconducting transition of $\mathrm{MgB}_{2}$ at $7 \mathrm{GPa}$.

*These authors contributed equally to this work. 
Compression of a solid directly changes its electron density inducing a large diversity of magnetic phenomena such as quantum critical points or high-spin low-spin transitions (1). Pressure is also a relevant physical parameter for tuning superconductivity in a wide range of systems, such as cuprates, transition-metal dichalcogenides, iron pnictides, heavy fermions or topological superconductors. Recently a novel class of high temperature superconductors has been discovered in H-rich hydrides at high pressure, highlighted by the reported critical temperature of $203 \mathrm{~K}$ in $\mathrm{H}_{3} \mathrm{~S}$ at $150 \mathrm{GPa}$ (2). Systematic exploration of these materials requires sensitive magnetic characterization that can be routinely operated in the $100 \mathrm{GPa}$ range (3).

Great efforts have already been made to adapt magnetic detection methods to the DAC specificities (4). The macroscopic magnetic susceptibility of the compressed sample can be measured using inductively coupled coils. Two strategies have been followed to circumvent the poorly scaling filling factor under pressure, detrimental for the detection sensitivity. One is to insert the detection coil inside the sample chamber (5) and the other to miniaturize the whole DAC to integrate it in a SQUID in order to benefit from the intrinsic high sensitivity of SQUID measurements (6). Synchrotron based methods, such as $\mathrm{x}$ ray magnetic circular dichroism (XMCD), $\mathrm{x}$ ray emission spectroscopy (XES), and nuclear resonant forward scattering (NRFS) are also widely used due to the development of focused and high brightness synchrotron $\mathrm{x}$ ray beams. By addressing atomic or nuclear resonance lines, these methods are element specific and resolve local magnetism due to a given electronic order (7). However, quantitative analysis exploiting these techniques may be challenging $(8,9)$. Furthermore, synchrotron-based techniques, mainly probing nuclear or electronic transitions, are not directly sensitive to magnetic phenomena such as the Meissner effect which is the signature of superconductivity.

We report an optical magnetometry method based on NV color centers used as in situ quantum sensors (Fig. 1a). The main advantages are the easiness of the sample preparation, the table-top instrumentation, the mapping of the stray magnetic field with micrometer spatial reso- 
lution and the absence of any sensitivity decrease with the sample size, down to the micrometer scale. Being non invasive, the scheme of this magnetic detection can be easily associated to complementary structural determination by $\mathrm{x}$ ray diffraction. The method is based on Optically Detected Magnetic Resonance (ODMR) which exploits the triplet fine structure of the negatively charged $\mathrm{NV}^{-}$center ground state (Fig. 1b). ODMR relies on the difference in luminescence intensity when exciting the $\mathrm{NV}^{-}$center from $m_{S}=0$ (high intensity) or from $m_{S}= \pm 1$ (low intensity) spin states (10). This property is used to perform a spectroscopy of the $m_{S}=0 \rightarrow \pm 1$ transition excited by a microwave (MW) signal which frequency is scanned across the resonance. Under a continuous MW excitation in the absence of external perturbation, the spin dependent luminescence recorded as a function of the MW frequency exhibits a resonance peak at $2.87 \mathrm{GHz}$, as shown in Fig. 1c. An external magnetic field applied on the NV center then splits this resonance, leading to the direct measurement of the magnetic field amplitude from the ODMR spectrum (11). The influence of strain on this transition was investigated up to a pressure of about $60 \mathrm{GPa}$ by Doherty et al and the application to magnetic detection in a DAC was envisioned as the main goal (12). Here, we fabricate the NV centers in the diamond anvil and we elaborate an analysis that unlocks magnetometry. We illustrate its efficiency on two testbed examples: a quantitative measurement of the magnetization of iron up to $30 \mathrm{GPa}$ under ambient temperature around the $\alpha \rightarrow \epsilon$ phase transition (13) and the detection of the superconductivity of $\mathrm{MgB}_{2}$ at $7 \mathrm{GPa}$ and $30 \mathrm{~K}(14)$.

A focused ion beam extracted from a nitrogen plasma is used to create the NV centers at the culet of an ultra-pure synthetic (IIas) diamond anvil (15), with a layer of about $10^{4}$ defects per $\mu \mathrm{m}^{2}$ at a depth of $20 \mathrm{~nm}$ below the surface of the anvil culet (see Supporting Information). As shown in Fig. 1a, the optical excitation of these shallow NV centers using a laser of $532 \mathrm{~nm}$ wavelength and the detection of their luminescence are performed through the anvil. We then implemented the wide-field ODMR scheme of (16) to record the map of the NV centers spin- 
state dependent luminescence as a function of microwave frequency. The implanted diamond anvil is mounted on a membrane DAC and a rhenium gasket laterally confines the sample. A single turn coil is positioned on the gasket as a MW antenna for the ODMR (Fig. 1a). A ruby crystal is used as a pressure gauge. Microscopic samples of iron or $\mathrm{MgB}_{2}$ were positioned in the sample chamber, directly in contact with the implanted anvil (see Fig. 2), and are embedded in a pressure transmitting medium consisting respectively of nitrogen and argon.

In the case of iron, various samples have been loaded to test geometric and size effects. The detailed analysis focuses on one of the iron beads (see Supporting Information for the signals associated to the other samples). A magnetic field $B_{0} \approx 11 \mathrm{mT}$, created by a permanent magnet, is applied to magnetize the iron samples and to split and resolve the resonances associated to the four orientations of the NV centers existing in a [100]-oriented diamond (17).

The energy levels of a given NV center are modified by the magnetic field and by the strain field existing in the anvil. The combination of these two perturbations results in both a shift and a splitting of the MW resonance frequency (18). The combined effect of strain and magnetic field has been described by Barson et al (19). As sketched in Fig. 1b, the hydrostatic component of the strain shifts the resonance frequency whereas its non-hydrostatic component and the magnetic field both split the resonance around its center frequency. Extracting the magnetic field created by the iron bead magnetization from these resonances recorded at high pressure requires to take into account the competing effects of the magnetic field and of the strain field which add quadratically (19). A typical spectrum obtained in the experiment is shown in Fig. 1c. At first order, the effect of the magnetic field is proportional to its longitudinal component on the N-V axis. It then leads to a spectrum consisting of a set of four double resonances corresponding to the four NV orientations. A map of the measured raw frequency splittings for each family of NV centers is plotted in Fig. 2 at a pressure of $24 \mathrm{GPa}$. The splitting induced by the iron bead magnetization is of the order of a few $\mathrm{MHz}$, which can be resolved over the splitting induced 
by $B_{0}$. Even prior to any data analysis, such images can be recorded live during the experiment providing direct evidence of pressure induced modifications of the magnetic properties.

After ascribing each pair of resonances to a given [111] axis of the diamond anvil, the stray magnetic field created by the iron bead can be quantitatively extracted from the correlated informations that are embedded in the spectrum shown in Fig. 1c (20). Beforehand, the strain component is extracted from the ODMR signal to sort out the stray magnetic field of the iron bead by using a reference area of the image selected far from the bead. It is also observed that right below the bead, the NV centers undergo a strong transverse magnetic field which mixes the $m_{S}= \pm 1$ states and induces a strong decrease of the ODMR contrast (21). This quenching leads to a non-relevant background signal without a direct link to the iron bead magnetization. As shown in Fig. 3b, the area below the center of the bead is excluded by applying a mask to the data according to a threshold set on the contrast (see Supporting Information for the data processing). At high pressure where the bead magnetization decreases, the masked area decreases accordingly.

The magnetization $M$ of the iron bead is then determined by fitting the relevant part of the magnetic field map with a simple magnetic dipole model (see Supporting Information). At low pressure, we obtain $M \approx 8 \pm 1 \mathrm{kA} \cdot \mathrm{m}^{-1}$. The evolution of the magnetization with pressure is shown in Fig. 3a. The magnetic field of the iron bead decreases as the pressure increases from $15 \mathrm{GPa}$ up to about $30 \mathrm{GPa}$ (Fig. 3b), above which no significant value of the magnetization can be inferred. This result demonstrates the sensitivity of the detection scheme and is consistent with previous XES and SQUID measurements that reported magnetic signals well above the $\alpha-\epsilon$ transition threshold $(22,23)$. Whether a remaining of the $\alpha$ phase is responsible for this magnetic signature up to $30 \mathrm{GPa}$ could be investigated by implementing NV-based magnetometry on an $\mathrm{x}$ ray diffraction beamline, combining structural and magnetic measurements on the same sample at each pressure. Finally, the pressure in the DAC was 
released; we then observed the reappearing of the sample magnetization with the expected hysteresis behavior related to the hysteresis of the structural transition (13).

This technique can be straightforwardly implemented at low temperatures in order to observe the Meissner effect associated to a superconductor. As a testbed system, we chose a sample of $\mathrm{MgB}_{2}$ that was confined in the DAC at $7 \mathrm{GPa}$ and first cooled in a cryostat in the absence of external magnetic field. At a temperature of about $18 \mathrm{~K}$, an external magnetic field $\approx 1.8 \mathrm{mT}$ is applied. The direction of this magnetic field is chosen parallel to the [100] diamond axis so that the four NV orientations in the crystal have identical responses. As shown in Fig. 4a, the comparison between the frequency shift in the electron spin resonance (ESR) of the NV centers located above the sample and the homogeneous background gives a direct image of the Meissner effect then providing a direct proof of $\mathrm{MgB}_{2}$ superconductivity (Fig. 4a). Under heating of the DAC, vanishing of the superconductivity is detected above $30 \mathrm{~K}$ (Fig. 4b). This behavior agrees with the reported pressure evolution of the $\mathrm{MgB}_{2}$ critical temperature (14).

The next step is to investigate how this direct detection of the Meissner effect can be implemented at a pressure range above $100 \mathrm{GPa}$. This may require adapting the excitation and readout wavelengths of the NV center in order to compensate for the pressure induced energy shifts of the electronic levels (12). NV engineering based on controlled nitrogen doping during the plasma-assisted growth of a diamond layer (24) or using laser writing (25) can be used to bury a thin sheet of NV centers at a depth where the influence of strain in the anvil could be less detrimental. A foreseen major application is the investigation of high-temperature superconductivity in the various super-hydride compounds that can be directly synthesized at high pressure, such as $\mathrm{H}_{3} \mathrm{~S}(2), \mathrm{UH}_{7}(26), \mathrm{LaH}_{10}(27)$ and $\mathrm{FeH}_{5}$ (28). Finally, it could enable the observation of the predicted magnetic properties of metallic hydrogen which is within experimental reach (29) and for which various challenging experimental probes have already been proposed $(30,31)$. 


\section{References and Notes}

1. H. K. Mao, X.J. Chen, Y. Ding, B. Li, and L. Wang, Solids, liquids, and gases under high pressure. Rev. Mod. Phys. 90, 015007 (2018).

2. A. P. Drozdov, M. I. Eremets, I. A. Troyan, V. Ksenofontov, and S. I. Shylin, Conventional superconductivity at 203 kelvin at high pressures in the sulfur hydride system. Nature 525, 73-76 (2015).

3. L. P. Gorkov and V. Z. Kresin, Colloquium: High pressure and road to room temperature superconductivity. Rev. Mod. Phys. 90, 011001 (2018).

4. M. I. Eremets, High-pressure experimental methods. Oxford University Press (New York, 1996).

5. D. D. Jackson, C. Aracne-Ruddle, V. Malba, and S. T. Weir, Magnetic susceptibility measurements at high pressure using designer diamond anvils. Rev. Sci. Instrum. 74, 2467 (2003).

6. A. Marizy, B. Guigue, F. Occelli, B. Leridon, and P. Loubeyre, A symmetric miniature diamond anvil cell for magnetic measurements on dense hydrides in a SQUID magnetometer. High Press. Res. 37, 465-474 (2017).

7. R. Torchio, Y. O. Kvashnin, S. Pascarelli, O. Mathon, C. Marini, L. Genovese, P. Bruno, G. Garbarino, A. Dewaele, F. Occelli, and P. Loubeyre, X-ray magnetic circular dichroism measurements in Ni up to $200 \mathrm{GPa}$ : Resistant ferromagnetism. Phys. Rev. Lett. 107, 237202 (2011).

8. J. P. Rueff and A. Shukla, Inelastic x-ray scattering by electronic excitations under high pressure. Rev. Mod. Phys. 82, 847-896 (2010). 
9. O. Mathon, F. Baudelet, J.-P. Itié, S. Pasternak, A. Polian, and S. Pascarelli, XMCD under pressure at the Fe k edge on the energy-dispersive beamline of the ESRF'. J. Synchrotron Rad. 11, 423-427 (2004).

10. M. W. Doherty, N. B. Manson, P. Delaney, F. Jelezko, J. Wrachtrup , and L. C. L. Hollenberg, The nitrogen-vacancy colour centre in diamond. Phys. Rep. 528, 1-45 (2013).

11. J. M. Taylor, P. Cappellaro, L. Childress, L. Jiang, D. Budker, P.R. Hemmer, A. Yacoby, R.L. Walsworth, and M.D. Lukin, High-sensitivity diamond magnetometer with nanoscale resolution. Nat. Phys. 4, 810-816 (2008).

12. M. W. Doherty, V. V. Struzhkin, D. A. Simpson, L. P. McGuinness, Y. Meng, A. Stacey, T. J. Karle, R. J. Hemley, N. B. Manson, L. C. L. Hollenberg, and S. Prawer, Electronic properties and metrology applications of the diamond NV center under pressure. Phys. Rev. Lett. 112, 47601 (2014).

13. W. A. Bassett and E. Huang, Mechanism of the body-centered cubic-hexagonal closepacked phase transition in iron. Science 238, $780-783$ (1987).

14. C. Buzea and T. Yamashita, Review of the superconducting properties of $\mathrm{MgB}_{2}$. Supercond. Sci. Technol. 14, R115-R146 (2001).

15. M. Lesik, P. Spinicelli, S. Pezzagna, P. Happel, V. Jacques, O. Salord, B. Rasser, A. Delobbe, P. Sudraud, A. Tallaire, J. Meijer, and J.-F. Roch, Maskless and targeted creation of arrays of colour centres in diamond using focused ion beam technology. Phys. Status Solidi A 210, 2055-2059 (2013).

16. S. Steinert, F. Dolde, P. Neumann, A. Aird, B. Naydenov, G. Balasubramanian, F. Jelezko, and J. Wrachtrup, High sensitivity magnetic imaging using an array of spins in diamond. Rev. Sci. Instrum. 81, 043705 (2010). 
17. L. Toraille, K. Azel, E. Balloul, C. Vicario, C. Monzel, M. Coppey, E. Secret, J. M. Siaugue, J. Sampaio, S. Rohart, N. Vernier, L. Bonnemay, T. Debuisschert, L. Rondin, J.-F. Roch, and M. Dahan, Optical magnetometry of single biocompatible micromagnets for quantitative magnetogenetic and magnetomechanical assays. Nano Lett. 18, 7635-7641 (2018).

18. J. Teissier, A. Barfuss, P. Appel, E. Neu, and P. Maletinsky, Strain coupling of a nitrogenvacancy center spin to a diamond mechanical oscillator. Phys. Rev. Lett.113, 020503 (2014).

19. M. S. J. Barson, P. Peddibhotla, P. Ovartchaiyapong, K. Ganesan, R. L Taylor, M. Gebert, Z. Mielens, B. Koslowski, D. A. Simpson, L. P McGuinness, J. McCallum, S. Prawer, S. Onoda, T. Ohshima, A. C. Bleszynski Jayich, F. Jelezko, N. B Manson, and M. W. Doherty, Nanomechanical sensing using spins in diamond. Nano Lett. 17, 1496-1503 (2017).

20. M. Chipaux, A. Tallaire, J. Achard, S. Pezzagna, J. Meijer, V. Jacques, J.-F. Roch, and T. Debuisschert, Magnetic imaging with an ensemble of nitrogen-vacancy centers in diamond. Eur. Phys. J. D 69, 166 (2015).

21. J.-P. Tetienne, L. Rondin, P. Spinicelli, M. Chipaux, T. Debuisschert, J.-F. Roch, and V. Jacques, Magnetic-field-dependent photodynamics of single NV defects in diamond: an application to qualitative all-optical magnetic imaging'. New J. Phys. 14, 103033 (2012).

22. A. Monza, A. Meffre, F. Baudelet, J.-P. Rueff, M. d?Astuto, P. Munsch, S. Huotari, S. Lachaize, B. Chaudret, and A. Shukla, Iron under pressure: Kohn tweezers and remnant magnetism. Phys. Rev. Lett. 106, 247201 (2011).

23. Q. Wei, C. McCammon, and S. A. Gilder, High-pressure phase transition of iron: a Combined magnetic remanence and Mossbauer study. Geochem. Geophys. Geosys. 18, 4646-4654 (2017). 
24. M. Lesik, N. Raatz, A. Tallaire, P. Spinicelli, R. John, J. Achard, A. Gicquel, V. Jacques, J.-F. Roch, J. Meijer, and S. Pezzagna, Production of bulk NV centre arrays by shallow implantation and diamond CVD overgrowth. Phys. Status Solidi A 213, 2594-2600 (2016).

25. Y.-C. Chen, P. S. Salter, S. Knauer, L. Weng, A. C. Frangeskou, C. J. Stephen, S. N. Ishmael, P. R. Dolan, S. Johnson, B. L. Green, G. W. Morley, M. E. Newton, J. G. Rarity, M. J. Booth, and J. M. Smith, Laser writing of coherent colour centres in diamond. Nature Phot. 11, 77 (2017).

26. I. A. Kruglov, A. G. Kvashnin, A. F. Goncharov, A. R. Oganov, S. S. Lobanov, N. Holtgrewe, S. Jiang, V. B. Prakapenka, E. Greenberg, and A. V. Yanilkin, Uranium polyhydrides at moderate pressures: Prediction, synthesis, and expected superconductivity. Science Adv. 12, eaat9776 (2018).

27. Z. Geballe, H. Liu, A. K. Mishra, M. Ahart, M. Somayazulu, Y. Meng, M. Baldini, and R. J. Hemley, Synthesis and stability of lanthanum superhydrides. Angew. Chem. Int. Ed. 57, 688-692 (2018).

28. C. M. Pépin, G. Geneste, A. Dewaele, M. Mezouar, and P. Loubeyre, Synthesis of FeH${ }_{5}$ : A layered structure with atomic hydrogen slabs. Science 357, 382-385 (2017).

29. R. P. Dias and I. F. Silvera, Observation of the Wigner-Huntington transition to metallic hydrogen. Science 355, 715-718 (2017). See comments by X.-D. Liu et al., Science 357, eaan2286 (2017) and A.F. Goncharov and V. V. Struzhkin Science 357, eaam9736 (2017).

30. E. Babaev, A. Sudb, and N. W. Ashcroft, Observability of a projected new state of matter: a metallic superfluid. Phys. Rev. Lett. 95, 105301 (2005).

31. J. P. Carbotte, E.J. Nicol, and T. Timusk, Detecting superconductivity in the high pressure hydrides and metallic hydrogen from optical properties. Phys. Rev. Lett. 121, 047002 (2018). 
32. We thank Vincent Jacques, Koen De Hantsetters, Nathalie Vast and Frédéric Bouquet for fruitful discussions. This work is supported by the EU FET-OPEN Flagship Project ASTERIQS (grant 820394), the Agence Nationale de la Recherche (project ENVIE-FIB), the Investissements dAvenir program through the LabEx PALM (ANR-10-LABX-0039-PALM), the Paris-Saclay Strategic Research Initiative for Quantum Engineering (IQUPS), and the Paris Île-de-France Région in the framework of DIM SIRTEQ. 


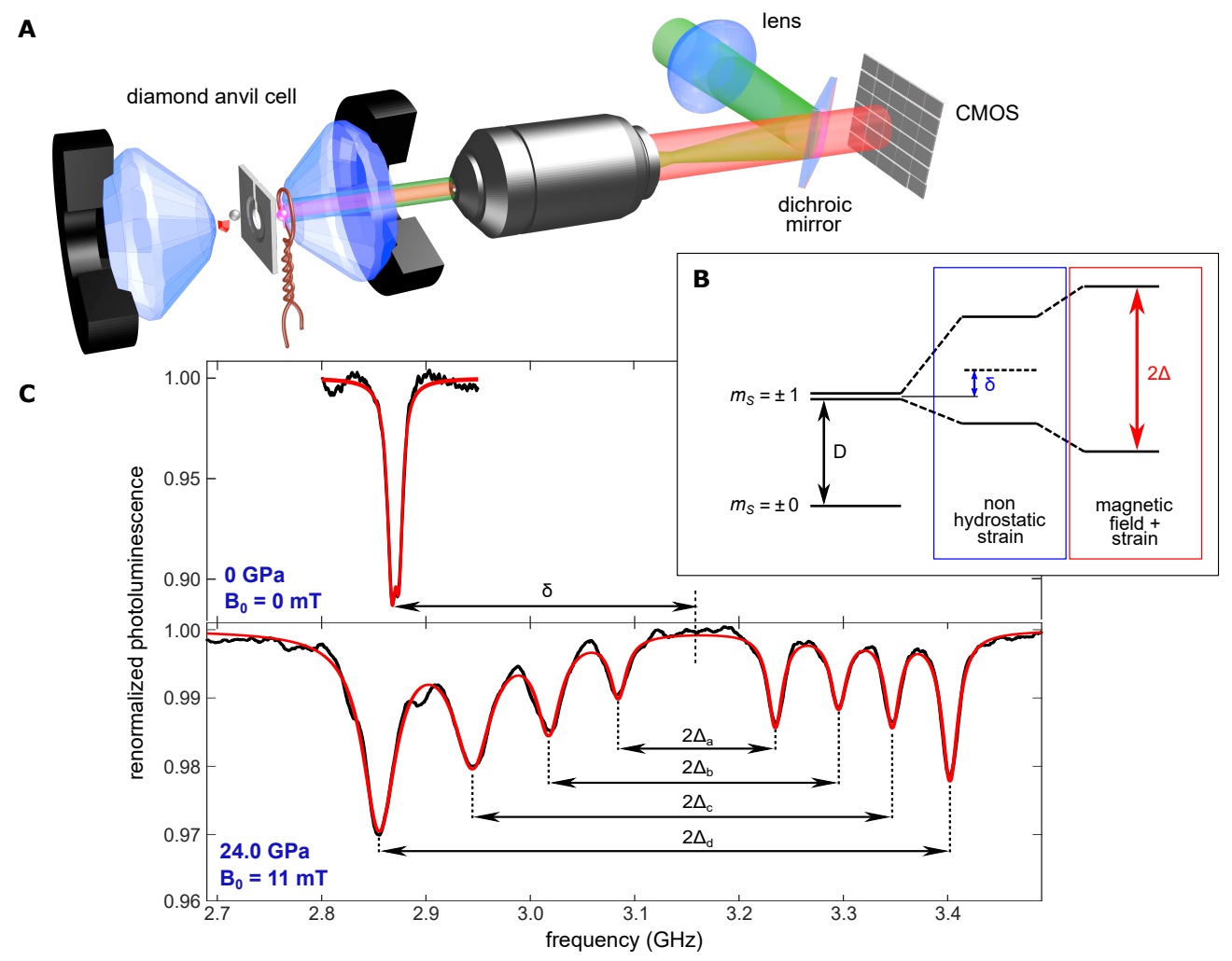

Fig. 1. Implementation of NV magnetometry in a DAC. (A) Scheme of the DAC setup. A $250 \mu \mathrm{m}$-wide disk of NV centers is implanted in the $300 \mu \mathrm{m}$-wide culet of one of the two anvils. A rhenium gasket encloses the sample and a ruby pressure gauge. A single-loop wire is placed on the gasket to generate the microwave excitation. A laser with $532 \mathrm{~nm}$ wavelength is used to excite the luminescence of the NV centers. The electron spin resonance is detected through its effect on the luminescence by using a CMOS camera. (B) Electronic structure of the NV center ground state with the modification of the energy levels induced by the strain in the anvil and the magnetic field. (C) Typical resonance spectra. In the absence of any perturbation the spectrum consists of a single resonance at $\mathrm{D}=2.87 \mathrm{GHz}$. Hydrostatic compression shifts this resonance by $\delta$ while non-hydrostatic strain splits the resonance in two components that are also affected by the applied magnetic field with a total frequency splitting $2 \Delta$. The projections of the magnetic field on the four NV orientations in the crystal lead to eight resonance peaks. 


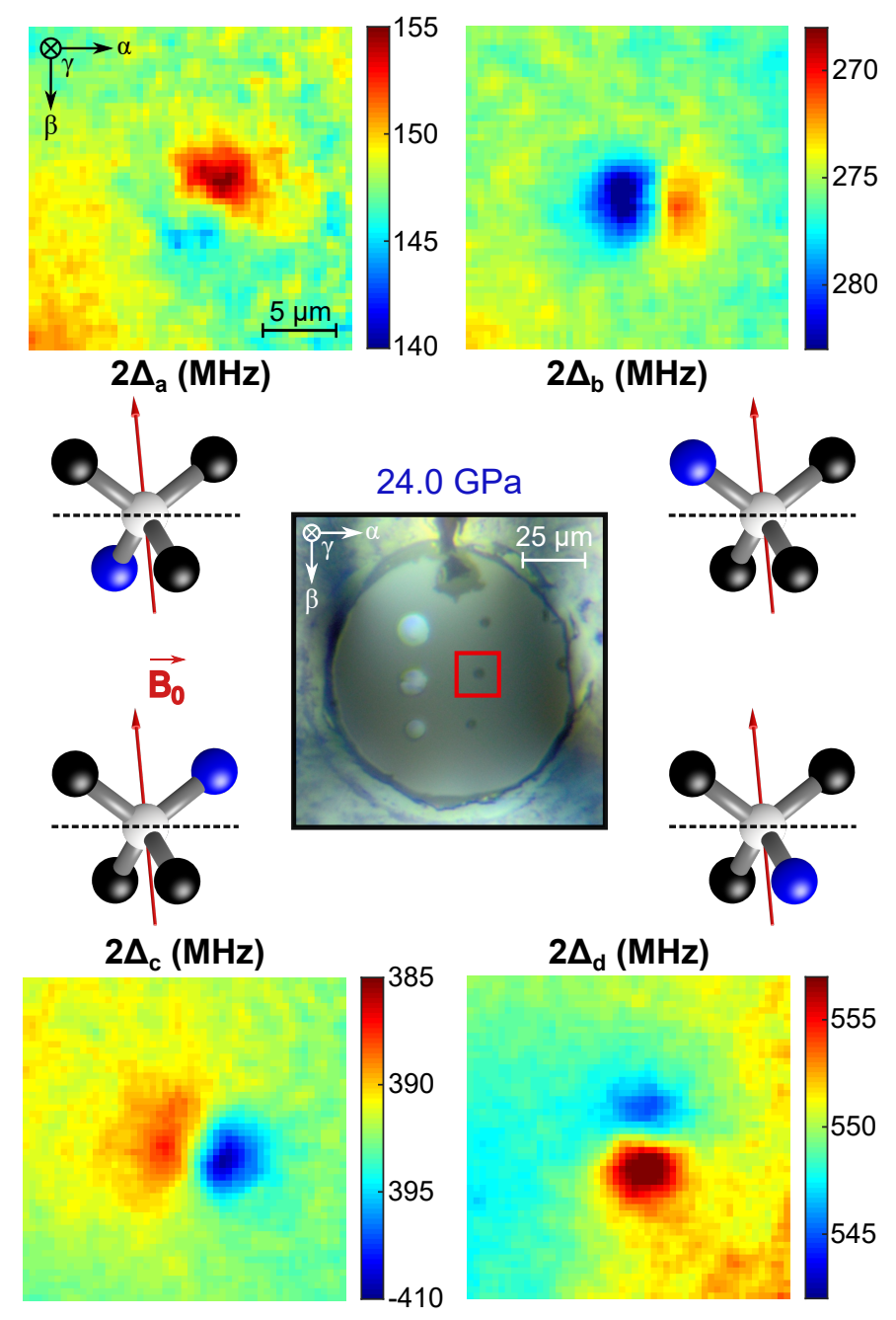

Fig. 2. Frequency splittings associated to the magnetization of the iron bead at 24 GPa for the four NV orientations. The data are shown for the bead indicated by the red square in the center image showing the iron samples inside the gasket. The splittings combine the effect of non hydrostatic strain in the anvil, of the applied magnetic field and of the stray magnetic field created by the bead magnetization. $(\alpha, \beta, \gamma)$ are reference axes linked to the anvil used to identify the four NV orientations. The dotted lines indicate the orientation of the anvil surface. 


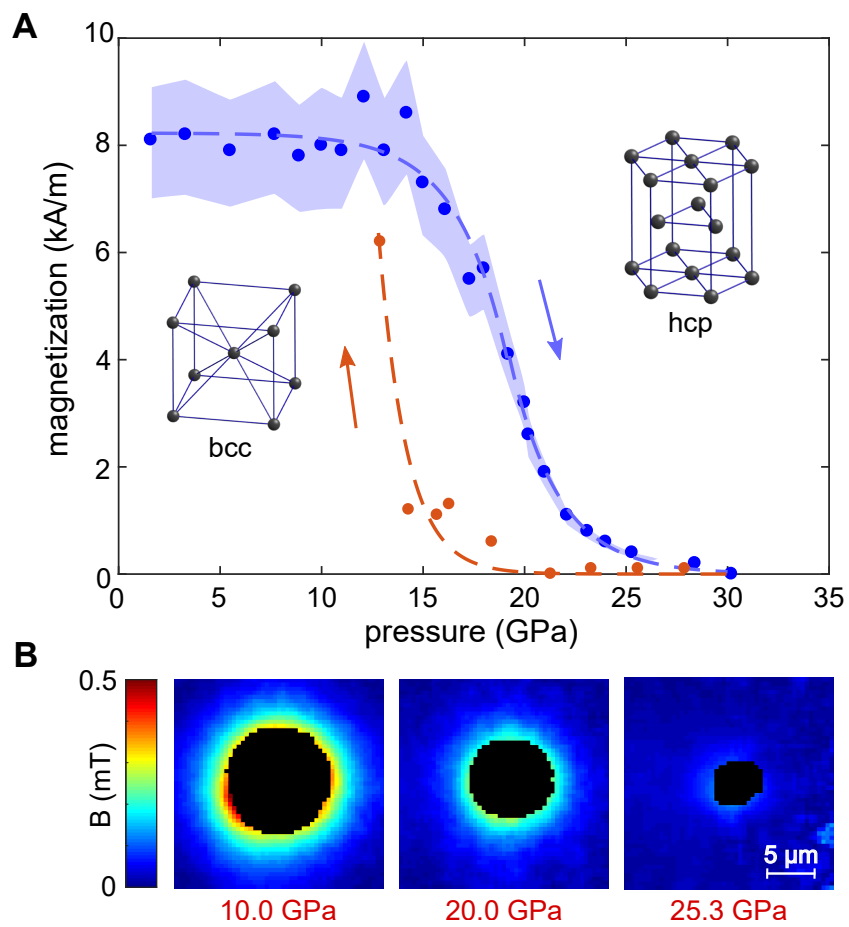

Fig. 3. Observation of the $\alpha-\epsilon$ transition of iron associated to (bec) and (hep) crystal structures. (A) Evolution of the bead magnetization inferred at each pressure. The data taken during the pressure increase is shown by blue dots while the data taken during the pressure release is shown by red dots. The shaded area shows the uncertainty interval on the magnetization value during the pressure increase. The dotted lines are simple guides to the eye. (B) Evolution of the amplitude of the magnetic field created by the iron bead. The mask shown in black is associated to the criteria set on the ODMR contrast. 
A
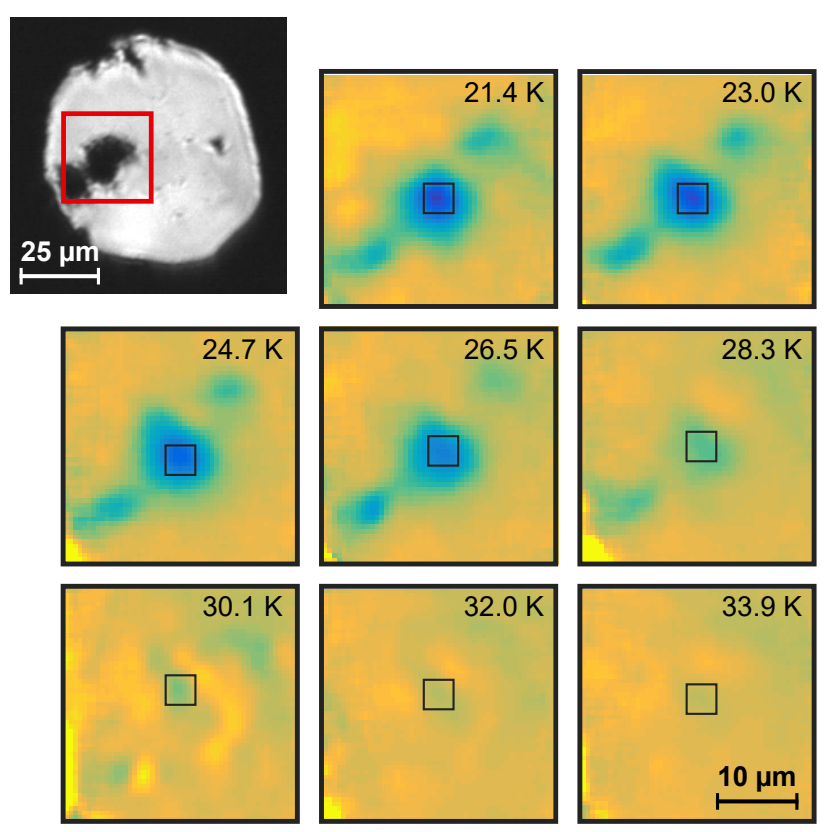

$\square$

$10 \mu \mathrm{m}$
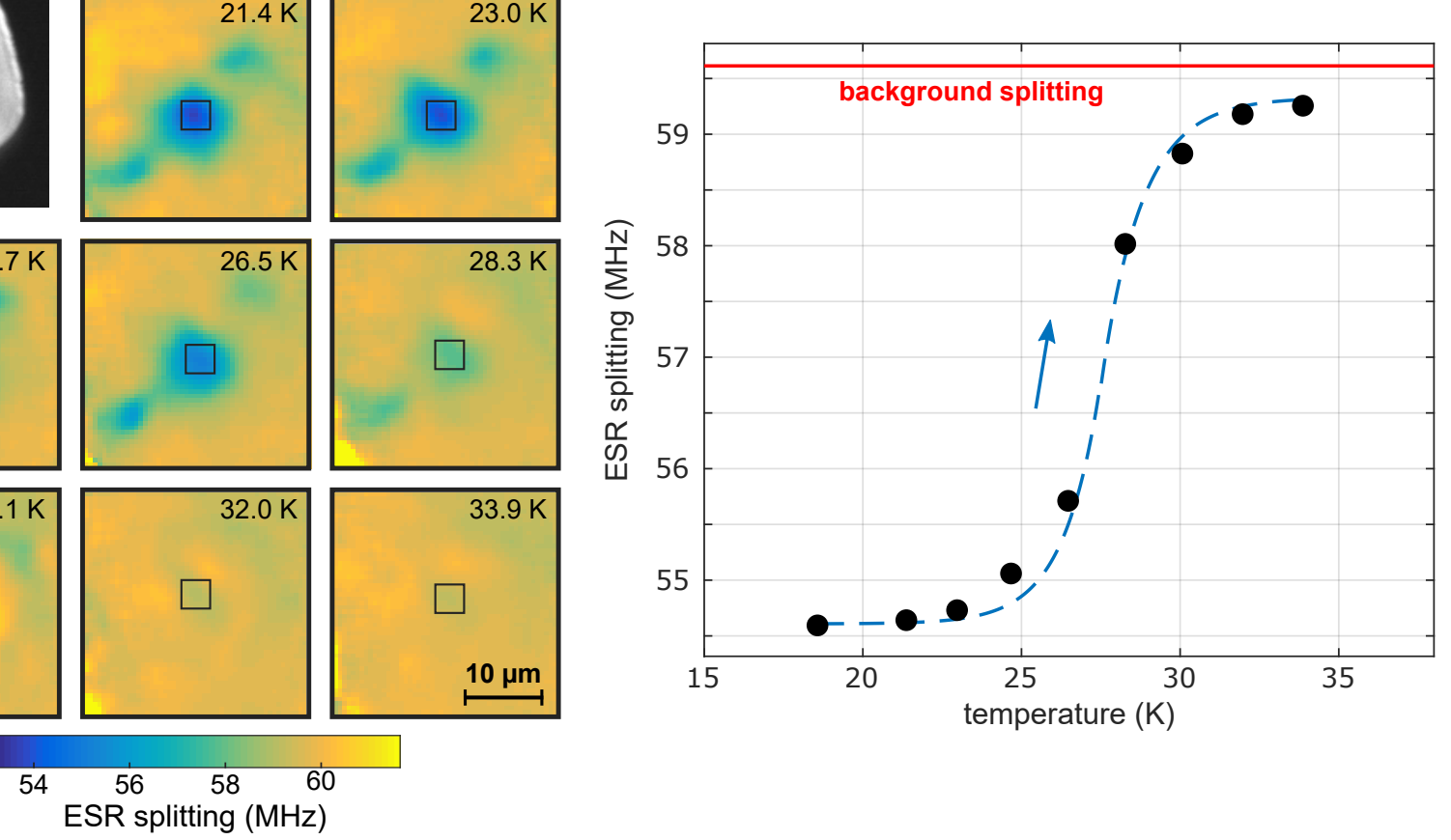

B

Fig. 4. Meissner effect associated to the superconductivity of $\mathrm{MgB}_{2}$ at 7 GPa. (A) Maps of the ESR frequency splitting above the $\mathrm{MgB}_{2}$ sample recorded for an increasing temperature. A control magnetic field $B_{0} \approx 1.8 \mathrm{mT}$ is applied and induces a background ESR splitting which combines the influence of $B_{0}$ with the strain in the anvil. Below $30 \mathrm{~K}$, the exclusion of the magnetic field is observed above the $\mathrm{MgB}_{2}$ sample due to the Meissner effect. This effect disappears above $30 \mathrm{~K}$ leading to an homogeneous distribution of the ESR splitting. Inset: optical image of the sample. The red square indicates where the ESR splitting is mapped. (B) Evolution of the ESR splitting above the sample when the temperature is increased. The data points are averaged on the black squares of $(\mathbf{A})$. The dotted line is a guide to the eye. 